Pure and Applied Mathematics Quarterly

Volume 1, Number 4

(Special Issue: In Memory of

Armand Borel, Part 3 of 3 )

$737-754,2005$

\title{
Construction of Some Generalised Modular Symbols
}

\author{
B. Speh and T. N. Venkataramana
}

\begin{abstract}
We give a criterion for the non-vanishing of certain modular symbols on a locally symmetric manifold. The criterion is in terms of the non-vanishing of some cohomology classes on the compact dual of the locally symmetric manifold. Using this, we construct nonzero modular symbols for $S L_{2 n}$ over an imaginary quadratic extension of $\mathbb{Q}$, which represent ghost classes. We also construct nonzero modular symbols in certain non-compact Shimura varieties and give an example of a modular symbol that generates an infinite dimensional module under the action of the Hecke algebra.
\end{abstract}

\section{INTRODUCTION}

Let $G$ and $H$ be semi-simple algebraic groups over $\mathbb{Q}$ and $f: H \rightarrow G$ a morphism with finite kernel of $\mathbb{Q}$-algebraic groups. Let $K_{H}$ be a maximal compact subgroup of the group $H(\mathbb{R})$ of real points of $H$ and $K \supset f\left(K_{H}\right)$ a maximal compact subgroup of $G(\mathbb{R})$. We then get a map $Y \rightarrow X$ of the associated symmetric spaces $Y=H(\mathbb{R}) / K_{H}$ and $X=G(\mathbb{R}) / K$. Suppose that $X$ and $Y$ are of dimension $D$ and $d$ respectively.

Assume that $\Gamma \subset G(\mathbb{Q})$ is a torsion-free congruence subgroup. Then $S(\Gamma)=$ $\Gamma \backslash X$ is a manifold with finite volume under the $G(\mathbb{R})$ invariant metric (and volume form ) on $X$. Write $\Gamma \cap H$ for $f^{-1}(\Gamma)$ and $S_{H}(\Gamma)=\Gamma \cap H \backslash Y$. We have an immersion $S_{H}(\Gamma) \rightarrow S(\Gamma)$ induced by the map $f$. This identifies $S_{H}(\Gamma)$ with a closed subspace of $S(\Gamma)$. This follows as in [A] 2.7. For ease of notation, we will assume that the groups $G(\mathbb{R})$ and $H(\mathbb{R})$ are connected. Then, both the symmetric spaces $X$ and $Y$ are orientable with an orientation fixed by $G(\mathbb{R})$ and $H(\mathbb{R})$ respectively. This in turn makes $S(\Gamma)$ and $S_{H}(\Gamma)$ orientable.

Received July 17, 2005.

1991 Mathematics Subject Classification. Primary 11F75; Secondary 11F80 
Now, compactly supported cohomology classes in $H_{c}^{d}(S(\Gamma))$ may be pulled back to $S_{H}(\Gamma)$ and integrated on $S_{H}(\Gamma)$. Since $H_{c}^{d}\left(S_{H}(\Gamma)\right)=\mathbb{C}$ integration on $S_{H}(\Gamma)$ defines a linear form on $H_{c}^{d}(S(\Gamma))$ which may be thought of -by Poincaré dualityas an element $\left[S_{H}(\Gamma)\right]$ of $H^{D-d}(S(\Gamma))$. It is called the generalized modular symbol corresponding to $H$.

In the arithmetic of modular curves and classical automorphic forms modular symbols have served as an indispensable tool linking geometry and arithmetic. "Period integrals" of Eisenstein classes or cuspidal cohomology classes over compact modular symbols have been used by G.Harder to obtain information about special values of L-functions [H1], [H2]. In 1990 A. Ash and A.Borel showed that the Levi factors of parabolic subgroups define nonzero modular symbols [A-B], [R-S]. Later Ash, Ginzburg and Rallis give 6 families of pairs $(\mathrm{G}, \mathrm{H})$ where they can show that that any cuspidal cohomology class for $\Gamma$ over a generalized modular symbol corresponding to $\mathrm{H}$ vanishes [A-G-R]. One such pair is $G=S p_{n}$ and $H=S p_{m} \times S p_{k}$ which we also consider in theorem 3 .

In this paper we give a criterion for the non-vanishing of a modular symbol $\left[S_{H}(\Gamma)\right]$. The criterion is in terms of the compact dual spaces $\hat{X}$ and $\hat{Y}$ of the symmetric spaces $X$ and $Y$. We recall the construction of $\hat{X}$ and $\hat{Y}$; there is a Cartan decomposition of the Lie algebra $\mathfrak{g}_{0}$ of $G(\mathbb{R})$ with respect to the maximal compact $K$, which leaves the Lie algebra $\mathfrak{h}_{0}$ of $H(\mathbb{R})$ stable. Write $\mathfrak{g}_{0}=\mathfrak{k}_{0} \oplus \mathfrak{p}_{0}$ and $\mathfrak{h}_{0}=\mathfrak{k}_{0} \cap \mathfrak{h}_{0} \oplus \mathfrak{p}_{0} \cap \mathfrak{h}_{0}$ as the Cartan decompositions. Let $G_{u}$ be the compact subgroup of $G(\mathbb{C})$ with Lie algebra $\mathfrak{k}_{0} \oplus i \mathfrak{p}_{0}$. Then $\widehat{X}=G_{u} / K$. The compact dual $\widehat{Y}$ is defined analogously. One has an embedding of $\widehat{Y}$ in $\widehat{X}$, and the fundamental class $[\widehat{Y}]$ of $\widehat{Y}$ in $\widehat{X}$ is a cohomology class in $H^{D-d}(\widehat{X})$.

The Borel map $j$ from the cohomology $H^{*}(\widehat{X})$ into the cohomology $H^{*}(S(\Gamma))$ is defined as follows: we identify $H^{*}(\widehat{X})$ with $H^{*}(\mathfrak{g}, K, \mathbb{C})$ and $H^{*}(S(\Gamma))$ with $H^{*}\left(\mathfrak{g}, K, C^{\infty}(\Gamma \backslash G(\mathbb{R}))\right)$ where $\mathfrak{g}=\mathfrak{g}_{0} \otimes \mathbb{C}$; then the map $j$ is induced by the inclusion of the constant functions $\mathbb{C}$ in the space $C^{\infty}(\Gamma \backslash G(\mathbb{R}))$ of smooth functions on $\Gamma \backslash G(\mathbb{R})$.

With notation as above, we prove the following theorem in section 2 .

Theorem 1. Suppose that $G$ is a simply connected group which has no $\mathbb{R}$ anisotropic factors defined over $\mathbb{Q}$. Then, the class $j([\widehat{Y}])$ is a linear combination of Hecke translates of the generalised modular symbol $\left[S_{H}\left(\Gamma^{\prime}\right)\right] \in H^{D-d}\left(S\left(\Gamma^{\prime}\right)\right)$ for some congruence subgroup $\Gamma^{\prime}$ of $\Gamma$. In particular, if $j([\widehat{Y}]) \neq 0$, then the modular symbol $\left[S_{H}\left(\Gamma^{\prime}\right)\right]$ does not vanish.

The proof of this result relies on the work of J. Franke $[F]$. 
In section 3 we deduce, from Theorem 1 and some computations in the cohomology of classical compact symmetric spaces, the following theorem.

Theorem 2. If $E$ is a totally imaginary number field, $G=R_{E / \mathbb{Q}}\left(S L_{2 n}\right)$ and $H=R_{E / \mathbb{Q}}\left(S p_{2 n}\right)$, then the modular symbol $\left[S_{H}(\Gamma)\right]$ does not vanish for some congruence subgroup $\Gamma$.

If $E$ is a totally imaginary quadratic extension of a totally real number field $F$, $G=R_{E / \mathbb{Q}} S L_{2 n+1}$ and $H=R_{F / \mathbb{Q}} S L_{2 n+1}$, then the modular symbol $\left[S_{H}(\Gamma)\right]$ does not vanish for some congruence subgroup $\Gamma$.

In the above, $R$ denotes the (Weil) restriction of scalars.

A cohomology class was called a ghost class by A.Borel if it restricts trivially to each boundary component of the Borel Serre compactification,but its restriction to the full boundary is not zero. The first example of ghost class was constructed by G.Harder in the cohomology of $G L_{3}$ over totally imaginary fields using Eisenstein classes. Later a whole family of ghost classes was constructed by J. Franke using cohomology classes represented by invariant forms. We show

Corollary 1. The $G\left(\mathbb{A}_{f}\right)$-span of the modular symbols $\left[S_{H}(\Gamma)\right]$ in theorem 2 contains ghost classes.

Theorem 1 is especially useful in the case when the symmetric spaces $X$ and $Y$ are of Hermitian type and the embedding $Y \rightarrow X$ is holomorphic. In particular, we prove

Theorem 3. Let $G=S p_{2 g}$ be the split symplectic group over $\mathbb{Q}$ and let $H=$ $\prod S p_{2 g_{i}} \subset S p_{2 g}$ with $\sum g_{i}=g$, be the natural inclusion. Then, the modular symbol $\left[S_{H}(\Gamma)\right]$ is non-zero, for a suitable congruence subgroup $\Gamma$.

Analogously, for the unitary group, we prove

Theorem 4. Suppose $q \geq p \geq 1$ are integers, and let $G=U(p, q)$ be the unitary group in $p+q$ variables.

(1) If $p_{i}$ and $q_{i}$ are integers such that $\sum p_{i}=p$ and $\sum q_{i} \leq q$, and $H=$ $\prod U\left(p_{i}, q_{i}\right)$ then the modular symbol $\left[S_{H}(\Gamma)\right]$ is non-zero, for some congruence subgroup $\Gamma$ of $G$.

(2) Under the natural embedding of $H=S p_{2 g}$ in $G=U(g, g)$, the modular symbol $\left[S_{H}(\Gamma)\right]$ is non-zero for some congruence subgroup $\Gamma$.

In some instances, one can even determine if the space of the span of $G\left(\mathbb{A}_{f}\right)$ translates of the modular symbol $\left[S_{H}(\Gamma)\right]$ is infinite dimensional.

Theorem 5. Suppose that $G=U(1, q)$, and $H=U(1, r)$ with $r=q-2$ or $r=q-1$. Then there exists a congruence subgroup $\Gamma$ such that the $G\left(\mathbb{A}_{f}\right)$ translates of the modular symbol $\left[S_{H}(\Gamma)\right]$ is infinite dimensional. 


\section{Some Results of Franke and Proof of Theorem 1}

2.1. Notation. Let $\Gamma$ be as in Theorem 1. Let $K_{f}$ be the closure of $\Gamma$ in $G\left(\mathbb{A}_{f}\right)$, where $\mathbb{A}_{f}$ denotes the ring of finite adeles over $\mathbb{Q}$. Put $H^{*}\left(S_{G}\right)$ for the direct limit $\lim H^{*}(S(\Gamma))$ as $\Gamma$ varies over congruence subgroups of $G(\mathbb{Q})$ (if $\Gamma^{\prime} \subset \Gamma$, then there is a natural inclusion $H^{*}(S(\Gamma))$ in $H^{*}\left(S\left(\Gamma^{\prime}\right)\right)$ which gives us a direct system of finite dimensional complex vector spaces, and the direct limit is with respect to these inclusions). On this direct limit, the group $G\left(\mathbb{A}_{f}\right)$ operates, and if $K$ is the closure of $\Gamma$ in $G\left(\mathbb{A}_{f}\right)$ then, the space of $K_{f}$-invariants in $H^{*}\left(S_{G}\right)$ is exactly $H^{*}(S(\Gamma)$ ) (we are using strong approximation here which is guaranteed under the assumptions of Theorem 1$)$.

Let $K_{0}$ be a good maximal compact subgroup of $G\left(\mathbb{A}_{f}\right)$ (recall that this means that $K$ acts transitively on the quotient space $G\left(\mathbb{A}_{f}\right) / P\left(\mathbb{A}_{f}\right)$ for all parabolic $\mathbb{Q}$-subgroups of $G$ ). Put $\Gamma_{0}=G(\mathbb{Q}) \cap K_{0}$. We fix a subgroup $\Gamma^{\prime}$ of finite index in $\Gamma \cap \Gamma_{0}$. It is clear from the definition of the modular symbol $\xi_{\Gamma}=\left[S_{H}(\Gamma)\right]$ that the sum of translates of $\xi_{\Gamma^{\prime}}$ over a set of coset representatives of $\Gamma / \Gamma^{\prime}$ (resp. $\left.\Gamma_{0} / \Gamma^{\prime}\right)$ is a non-zero multiple of $\xi_{\Gamma}$ (resp. $\left.\xi_{\Gamma_{0}}\right)$. Therefore, if we show that $j([\widehat{Y}])$ is a linear combination of $G\left(\mathbb{A}_{f}\right)$-translates of $S_{H}\left(\Gamma_{0}\right)$ then we have proved Therem 1.

Set $\mathcal{H}_{0}$ to be the space of complex valued compactly supported $K_{0}$-bi-invariant functions on the finite adelic group $G\left(\mathbb{A}_{f}\right)$. This is an algebra (Hecke algebra corresponding to $\left.K_{0}\right)$ under convolutions and acts on the cohomology group $H^{*}\left(S\left(\Gamma_{0}\right)\right)=H^{*}\left(S_{G}\right)^{K_{0}}$. Let $\mathbb{C}$ denote the trivial one dimensional $G\left(\mathbb{A}_{f}\right)$-module. On this module, $\mathcal{H}_{0}$ operates, and we get a homomorphism $\chi: \mathcal{H}_{0} \rightarrow \mathbb{C}$. Let $\mathfrak{m}=\mathfrak{m}_{\chi}$ denote the kernel of the map $\chi$. This is a maximal ideal in $\mathfrak{H}_{0}$. Denote by $H^{*}\left(S_{G}\right)_{\mathfrak{m}}^{K_{0}}$ the space of vectors in $H^{*}\left(S_{G}\right)^{K_{0}}$ which are annihilated by some power of the ideal $\mathfrak{m}$. Clearly, this space is a direct summand as a Hecke module. We will refer to this space of vectors killed by the ideal $\mathfrak{m}$ as the space of "invariants".

However, the main theorem (Theorem 1 of $[\mathrm{F}]$ ) of $[\mathrm{F}]$ asserts that for the good maximal compact subgroup $K_{0}$, this space coincides with the space of vectors annihilated by the first power of the maximal ideal $\mathfrak{m}$ and that it is also isomorphic to the space of co-invariants for $G\left(\mathbb{A}_{f}\right)$ of the module $H^{*}\left(S_{G}\right)$. Moreover, according to $[\mathrm{F}]$, the latter is naturally isomorphic to $H^{*}(\mathcal{U})^{K \cap P(\mathbb{R})}$ where $P$ is a fixed minimal parabolic $\mathbb{Q}$-subgroup of $G$ and $\mathcal{U}$ is the following open set in the compact dual $\widehat{X}$ :

$$
\mathcal{U}=\widehat{X}-\cup \widehat{X_{L}}
$$

where the union is over Levi subgroups $L$ of parabolic $\mathbb{Q}$-subgroups of $G$ containing the minimal one $P$; $\widehat{X_{L}}$ denotes the compact dual of the symmetric space associated to the group $L^{0} \subset L(\mathbb{R})$. Here, $L^{0}$ is the subgroup of $L$, which is the 
intersection of the kernels of rational characters on $L$. The above isomorphism takes the restriction $\operatorname{res}([x])$ to $H^{*}(\mathcal{U})$ of a class $[x] \in H^{*}(\widehat{X})$, into the vector $j([x])$.

Now the space $V=H^{*}\left(S_{G}\right)^{K_{0}}$ is a finite dimensional complex vector space which is a module over the ring $\mathcal{H}_{0}$. Let $J$ be the kernel of the map $\mathcal{H}_{0} \rightarrow \operatorname{End}(V)$ and let $R=\mathcal{H}_{0} / J$ be the quotient ring (which is a finite dimensional algebra over $\mathbb{C})$. Then, $\mathfrak{m}$ is the inverse image of a maximal ideal $\mathfrak{m}_{R}$ of $R$ under the quotient map.

Let $I$ denote the annihilator in $R$ of the modular symbol $\left[S_{H}\left(\Gamma_{0}\right)\right]$. We may write $I=\mathfrak{m}_{R}^{k} A$ where $A$ is an ideal coprime to $\mathfrak{m}_{R}$. The span $W_{\left[S_{H}\left(\Gamma_{0}\right)\right]}$ of $\mathcal{H}_{0}$-translates of the class $\left[S_{H}\left(\Gamma_{0}\right)\right]$ is isomorphic as an $\mathcal{H}_{0}$ module, to $R / I=$ $R / \mathfrak{m}_{R}^{k} \oplus R / A$. By Franke's Theorem, if $k \geq 1$ then $k=1$. Consequently, if the projection $\operatorname{pr}\left(\left[S_{H}\left(\Gamma_{0}\right)\right]\right)$ of the modular symbol $\left[S_{H}\left(\Gamma_{0}\right)\right]$ to the above space of "invariants" (i.e. those annihilated by the ideal $\mathfrak{m}$ ) is non-zero, then the projection $\operatorname{pr}\left(\left[S_{H}\left(\Gamma_{0}\right)\right]\right)$ lies in $W_{\left[S_{H}\left(\Gamma_{0}\right)\right]}$ and hence it is a linear combination of Hecke translates of $\left[S_{H}\left(\Gamma_{0}\right)\right]$.

We will complete the proof of Theorem 1 by showing that this projection $\operatorname{pr}\left(\left[S_{H}\left(\Gamma_{0}\right)\right]\right)$ is a nonzero multiple of $j([\widehat{Y}])$.

The space of $\mathfrak{m}$ invariants is a direct summand of the $\mathcal{H}_{0}$-module $H^{*}\left(S\left(\Gamma_{0}\right)\right)$. We identify it as a vector space using the map $\mathrm{j}$ with $H^{*}(\mathcal{U})$. Hence under Poincare duality we can identify $H_{c}^{*}(\mathcal{U})$ with the space of $\mathfrak{m}$ invariants in $H_{c}^{*}\left(S\left(\Gamma_{0}\right)\right)$. Note that since the ring $\mathcal{H}_{0}$ is equipped with a nice involution induced from $g \mapsto g^{-1}$ on $G\left(\mathbb{A}_{f}\right)$, we can consider these spaces as dual modules of $\mathcal{H}_{0}$-modules.

For a given class $[w]$ in $j\left(H_{c}^{d}(\mathcal{U})\right)$ we consider the map

$$
W_{\left[S_{H}\left(\Gamma_{0}\right)\right]} \rightarrow H_{c}^{D}\left(S\left(\Gamma_{0}\right)\right)
$$

defined $[v] \rightarrow[v] \wedge[w]$ as a linear form on $W_{\left[S_{H}\left(\Gamma_{0}\right)\right]}$. Since $[\mathrm{w}]$ is $\mathcal{H}_{0}$-invariant this form is also $\mathcal{H}_{0}$-invariant and so it follows that for $[w] \in H_{c}^{d}(\mathcal{U})$, we have

$$
[v] \wedge[w]=\operatorname{pr}\left(\left[S_{H}\left(\Gamma_{0}\right)\right]\right) \wedge[w] .
$$

However the map

$$
H_{c}^{d}(\mathcal{U}) \rightarrow H_{c}^{D}\left(S_{G}\right)^{K_{0}}=j\left(H^{D}(\widehat{X})\right)
$$

given by wedging with the fundamental class $\left[S_{H}\left(\Gamma_{0}\right)\right]$ is up to a nonzero multiple the integral of $[w]$ over $\widehat{Y}$ and is hence equal to $)[\widehat{Y}] \wedge j^{*}\left([w]\right.$ where $j^{*}: H_{c}^{*}(\mathcal{U}) \rightarrow$ $H^{*}(\widehat{X})$ is the dual of the restriction map from the cohomology of $\widehat{X}$ to that of $\mathcal{U}$. 
By definition, this means that

$$
\left[S_{H}\left(\Gamma_{0}\right)\right] \wedge[w]=j([\widehat{Y}]) \wedge[w] .
$$

This holds for all $[w] \in H_{c}^{d}(\mathcal{U})$. Hence

$$
\operatorname{pr}\left(\left[S_{H}\left(\Gamma_{0}\right)\right]\right)=j([\widehat{Y}]) .
$$

Here, under the isomorphism of Franke mentioned in an earlier paragraph, the image of $j$ has been identified with the image of the restriction map from the cohomology of $\widehat{X}$ into that of $\mathcal{U}$.

This proves Theorem 1 .

2.2. Remark. The foregoing proof is an adaptation of $[\mathrm{V}]$ where the analogous result for the compact case is proved. In the compact case, the fact that the action by Hecke operators is completely reducible is used in a crucial way. The extension in the present paper to the non-compact case (i.e. when $S(\Gamma)$ is not compact) is achievable only because of the result of Franke that the space of Hecke algebra invariants is a direct summand -as a Hecke module- of $H^{*}\left(S_{G}\right)^{K_{0}}$ for the good maximal compact $K_{0}$.

\section{Applications}

In this section we apply the criterion of Theorem 1. Before doing so we note another result from $[F]$ ( see $[F],(7.1)$, equation (54)) on the kernel of the Borel map

$$
j: H^{*}(\widehat{X}) \rightarrow H^{*}(S(\Gamma)) .
$$

We have a dual map

$$
j^{*}: H_{c}^{*}(S(\Gamma)) \rightarrow H^{*}(\widehat{X}) .
$$

Franke's theorem says that a class $[x] \in H^{*}(\widehat{X})$ lies in the image of the compactly supported cohomology $H_{c}^{*}(S(\Gamma))$ if and only if the restriction of the class $[x]$ to $\widehat{X_{L}}$ vanishes for all Levi subgroups $L$ of all $\mathbb{Q}$-parabolic subgroups of $G$. Dually, this means that the kernel of the Borel map $j$ is the orthogonal complement of the space of vectors $[v] \in H^{*}(\widehat{X})$ whose restriction to $\widehat{X_{L}}$ vanishes for al the Levis $L$ as above. Here orthogonal complement means the following: the cohomology algebra $H^{*}(\widehat{X})$ comes equipped with a non-degenerate bilinear form (Poincare duality) and the orthogonal complement is with respect to this bilinear form.

3.1. Example. Let $G=R_{E / Q}\left(S L_{n}\right)$ with $E$ an imaginary quadratic extension of $\mathbb{Q}$. Then, $\widehat{X}=S U(n)$ and its cohomology is an exterior algebra

$$
\wedge=\wedge\left(e_{3}, e_{5}, \cdots, e_{2 n-1}\right)
$$

on primitive generators $e_{2 i-1}$ of degree $2 i-1$. 
Fix $k \geq 1$ and consider the inclusion $S U(k) \subset S U(n)$. The cohomology algebra of $S U(k)$ is the exterior algebra

$$
\wedge\left(e_{3}, e_{5}, \cdots, e_{2 k-1}\right)
$$

and the restriction map in the cohomology from $S U(n)$ to $S U(k)$ is given by $e_{i} \mapsto e_{i}$ if $i \leq 2 k-1$ and $e_{i} \mapsto 0$ otherwise (this is well known; e.g., see [M-T], Chapter (III), p.148, Theorem (6.5) (4)).

¿From the description of the Levi subgroups (they are of the form $R_{E / Q}\left(S L_{m_{1}} \times\right.$ $\left.\cdots \times S L_{m_{k}}\right)$ ) it follows that their compact duals are products of lower dimensional unitary groups. Using this and the definition of the generators $e_{2 i-1}$, it can be proved that a class $v \in \wedge$ restricts trivially to all these compact duals if and only if it is divisible by the class $e_{2 n-1}$. It is clear that the orthogonal complement of the ideal generated by $e_{2 n-1}$ is itself. Hence by Franke's theorem quoted at the beginning of this section, $j(v)=0$ if and only if $v$ is divisible by $e_{2 n-1}$.

Replace $n$ by $2 n$ and consider the embedding $H=R_{E / Q}\left(S p_{2 m}\right) \subset G=$ $R_{E / Q}\left(S L_{2 m}\right)$. The compact dual $\widehat{Y}$ is simply the group $S p_{2 n}$ whose cohomology is an exterior algebra

$$
\wedge\left(e_{3}, e_{7}, \cdots, e_{4 n-1}\right),
$$

on odd degree generators $e_{2 i-1}$ of degree $2 i-1$ with $i \geq 2$. The cohomology of $\widehat{X}$ is (note that $n$ is replaced by $2 n$ ) as we said before,

$$
\wedge\left(e_{3}, e_{5}, \cdots, e_{4 n-1}\right) .
$$

Moreover, the restriction map from $\widehat{X}$ to $\widehat{Y}$ takes $e_{i}$ to $e_{i}$ if $i=4 j-1$ and to 0 otherwise. This can easily be proved by looking more closely at the spectral sequences used to obtain the cohomology of $\widehat{X}$ and $\widehat{Y}$ (see [M-T], p.119, Ch. (III), Theorem (3.10) (1) and (2)).

Consequently, the fundamental class is

$$
[\widehat{Y}]=e_{5} \wedge e_{9} \wedge \cdots \wedge e_{4 n-3},
$$

and is not divisible by $e_{4 n-1}$. This proves that $j([\widehat{Y}]) \neq 0$. Therefore, the modular symbol $\left[S_{H}(\Gamma)\right]$ is non-zero for some congruence subgroup $\Gamma \subset G(\mathbb{Q})$.

Since $[\widehat{Y}]$ is not divisible by $e_{4 n-3}$, Franke's result shows that $j([\widehat{Y}])$ is not in the image of the cohomology with compact support. On the other hand the restriction of

$$
[\widehat{Y}]=e_{5} \wedge e_{9} \wedge \cdots \wedge e_{4 n-3}
$$

to the Levi subgroup $R_{E / Q}\left(S L_{n-1}\right)$ is in the kernel of the image of $\mathrm{j}$ for $R_{E / Q}\left(S L_{n-1}\right)$ since it is divisible by $e_{4 n-3}$. Thus $j([\widehat{Y}])$ is a ghost class. 
3.2. Example. Consider the embedding $S L_{2 n+1} \subset R_{E / \mathbb{Q}}\left(S L_{2 n+1}\right)$ of $\mathbb{Q}$-groups, where $E$ is an imaginary quadratic extension of $\mathbb{Q}$. The embedding $\widehat{Y}=S U(2 n+$ 1) $/ S O(2 n+1) \subset \widehat{X}=S U(2 n+1)$ is induced by $g \mapsto g g^{t}$ from $S U(2 n+1)$ into itself. The cohomology of $S U(2 n+1) / S O(2 n+1)$ is ( see [F], p. 35, Proposition 7) an exterior algebra

$$
\wedge\left(e_{5}, e_{9}, \cdots, e_{4 n+1}\right)
$$

and the restriction map from

$$
H^{*}(\widehat{X})=\wedge\left(e_{3}, e_{5}, \cdots, e_{4 n-1}, e_{4 n+1}\right)
$$

to $H^{*}(\widehat{Y})$ is given by sending $e_{2 i+1}$ to $e_{2 i+1}$ if $i$ is odd and to 0 otherwise.

Consequently, the fundamental class is

$$
[\widehat{Y}]=e_{3} \wedge e_{7} \wedge \cdots \wedge e_{4 n-1}
$$

and is therefore not divisible by $e_{4 n+1}$. Hence it does not lie in the kernel of the Borel map, and by Theorem 1 , the modular symbol $\left[S_{H}(\Gamma)\right]$ is non-zero for a suitable $\Gamma$.

The rest of Theorem 2 is proved in an entirely analogous way. The argument in the previous example shows that $j([\widehat{Y}])=j\left(e_{3} \wedge e_{7} \wedge \cdots \wedge e_{4 n-1}\right)$ is a ghost class.

3.3. Remark. Suppose that $H$ is any linear algebraic group over a totally real number field $F$ such that $H(F \otimes \mathbb{R})=S L_{2 n+1}(\mathbb{R})^{m}$ with $m$ the degree of $F$ over $\mathbb{Q}$ (that is, $H$ is a $F$-form of $\left.S L_{2 n+1}(\mathbb{R})^{m}\right)$. Let $E / F$ be a totally imaginary quadratic extension and set $G=R_{E / F}(H)$. Then, $\left[S_{H}(\Gamma)\right]$ is non-zero for some congruence subgroup of $G(E)$. This follows by the same method as in the case when $H=S L_{n}$ over $F$ (i.e. when $H$ is the standard $F$-form).

In the next two sections, we assume that both the symmetric spaces $X$ and $Y$ are Hermitian symmetric and that the embedding of $Y$ in $X$ is holomorphic. Under these assumptions, one can show in a large number of cases that the associated modular symbol $\left[S_{H}(\Gamma)\right]$ is non-zero for some $\Gamma$. We will discuss two important cases, when $G$ is the symplectic group $S p_{g}$ and the unitary group $U(p, q)$.

\section{The Symplectic Group}

In this section, $G=S p_{2 g}$ denotes the symplectic group defined and split over $\mathbb{Q}$. The associated symmetric space is the Siegel upper half space $X=S p_{2 g}(\mathbb{R}) / U(g)$. Denote by $\widehat{X}$ its compact dual. Let $T$ be the group of diagonal matrices in 
$K=U(g)$; it is a maximal torus in $K$ and in $G(\mathbb{R})$. The cohomology of the classifying space $B T$ is generated by Chern classes of line bundles arising from characters of $T$, and may be identified with the polynomial algebra $H^{*}(B T)=$ $\mathbb{C}[\Lambda]$, where $X^{*}(T)$ is the group of characters on $T$ and $\Lambda \subset X^{*}(T)$ is a suitable "positive subset" (in the case of $U(g)$ ), consisting of non-negative integral linear combination of the characters of $T$ occurring in the standard representation of $U(g)$ on $\mathbb{C}^{g}$. Clearly, the ring $R=\mathbb{C}[\Lambda]$ is $\mathbb{C}\left[x_{1}, \cdots, x_{g}\right]$ where $x_{i}$ is (by a mild abuse of notation) the Chern class of the character $x_{i}$ (the (i,i)-th entry) in the diagonal torus $T$. Note that the ring $R$ is a module for the Weyl groups $W_{G}=(\mathbb{Z} / 2 \mathbb{Z})^{g} \times S_{g}$ and $W_{K}=S_{g}$ of $G$ and $K$ respectively. Here $S_{g}$ is the symmetric group on the $g$ letters $x_{1}, x_{2}, \cdots, x_{g}$ and $W_{G}$ is a semi-direct product of $(\mathbb{Z} / 2 \mathbb{Z})^{g}$ with $S_{g}$; the elements of $W_{G}$ act on the $x_{i}$ by $\pm x_{\sigma(i)}$, with $\sigma \in S_{G}$. Let $\sigma_{1}, \cdots, \sigma_{g}$ denote the elementary symmetric functions in the variables $x_{1}, \cdots, x_{g}$.

Lemma 6. The cohomology of $\widehat{X}$ is the ring $\mathbb{C}\left[\sigma_{1}, \cdots, \sigma_{g}\right]$ modulo the ideal generated by the graded relation $\prod_{i}\left(1-x_{i}^{2}\right)=1$ ( $i$ runs from 1 to $g$ ).

Proof. The cohomology of the compact dual $\widehat{X}$ may be identified ([M-T]) with the ring $R^{W_{K}}$ of $W_{K}$-invariants in $R$ modulo the ideal generated by positive degree elements in the ring $R^{W_{G}}$ of $W_{G}$-invariants. The above description of the Weyl groups in question then implies the Lemma.

Next, we determine the kernel of the Borel map, using the criterion of Franke. Let $K=U(g) \subset \widehat{S p_{2 g}}$ be the natural inclusion, where $G_{u}=\widehat{S p_{2 g}}$ is the compact form of $G=S p_{2 g}$.

Lemma 7. If there is any embedding $i$ of the unitary group $U(g)$ in the compact form $\widehat{{S p_{2} g}_{1}}$ such that $K \cap i(S U(g))=O(g)$ the orthogonal group and the restriction of the embedding $i$ to $O(g)$ is identity, then, the restriction map from the cohomology (with $\mathbb{C}$-coefficients) of $\widehat{X}$ into that of the subsymmetric space $i(S U(g)) / S O(g)$ is zero except in degree zero.

Proof. To see this, first note that the cohomology of $\widehat{X}$ is generated by Chern classes of the homogeneous vector bundle arising from the standard representation of $U(g)$ on $\mathbb{C}^{g}$. Thus it is enough to show that these Chern classes vanish on the subsymmetric space. Now the restriction $E$ of this bundle to $i(S U(g)) / S O(g)$ is also homogeneous, and arises from the standard representation $\rho$ of $S O(g)$. Obviously, $\rho$ extends to a representation of $i(S U(g))$, which shows that the vector bundle $E$ admits a trivialisation. Hence all its higher degree Chern classes are zero. But these classes are the restriction of Chern classes of the vector bundle on $\widehat{\widehat{X}}$ with which we started. Therefore, the restriction of the cohomology of $\widehat{X}$ to $i(S U(g)) / S O(g)$ is trivial. 
Let $P \subset G$ be a standard maximal parabolic subgroup defined over $\mathbb{Q}$, and $L$ its Levi component. One may identify $L$ with $G L_{g-k} \times S p_{2 k}$ for some $k \leq g$. At the level of compact duals, one then has $\widehat{X_{L}}=S U(g-k) / S O(g-k) \times \widehat{S p_{2 k}} / U(k)$. Thus the restriction map from the cohomology of $\widehat{X}$ to $\widehat{X_{L}}$ factors through the product $\widehat{S p_{2 g}-2 k} / U(g-k) \times \widehat{S p_{2 k}} / U(k)$. From Lemma 7 (applied to $S p_{2 g-2 k}$ ) one sees that the kernel of the restriction map from $H^{*}(\widehat{X})$ to $H^{*}\left(\widehat{X_{L}}\right)$ is the same as the kernel of the restriction map from $H^{*}(\widehat{X})$ to $H^{*}\left(\widehat{S p_{2 k}} / U(k)\right)$.

Let $J$ be the kernel of the restriction map $H^{*}(\widehat{X}) \rightarrow \prod H^{*}\left(\widehat{X_{L}}\right)$ where the product runs over all the Levi subgroups $L$ of standard maximal parabolic $\mathbb{Q}$ subgroups $P$. Therefore, we have proved that the kernel of the foregoing restriction map is the same as the kernel of the map $H^{*}(\widehat{X}) \rightarrow H^{*}\left(\widehat{S p_{2 g-2}} / U(g-1)\right)$. Let $Z=\widehat{S p_{2 g-2}} / U(g-1)$, and let $\tau_{1}, \cdots, \tau_{g-1}$ denote the elementary symmetric functions in the $g-1$-variables $x_{2}, \cdots, x_{g}$. By Lemma 6 , we have the identifications

$$
H^{*}(\widehat{X})=\mathbb{C}\left[\sigma_{1}, \cdots, \sigma_{g}\right] / \prod_{i=1}^{i=g}\left(1-x_{i}^{2}\right)=1
$$

and

$$
H^{*}(Z)=\mathbb{C}\left[\tau_{1}, \cdots, \tau_{g-1}\right] / \prod_{i=2}^{i=g}\left(1-x_{i}^{2}\right)=1 .
$$

Lemma 8. The kernel of the Borel map $H^{*}(\widehat{X}) \rightarrow H^{*}(S(\Gamma))$ is orthogonal to the ideal generated by $\sigma_{g}$.

Proof. By Franke, the kernel of the Borel map is precisely the orthogonal complement (with respect to Poincaré duality on $\left.H^{*} \widehat{X}\right)$ ) of the kernel of the map $H^{*}(\widehat{X}) \rightarrow \prod H^{*}\left(\widehat{X_{L}}\right)$. By the discussion preceding the lemma, the latter is the kernel of the restriction map $H^{*}(\widehat{X}) \rightarrow H^{*}\left(\widehat{S p_{2 g-2}} / U(g-1)\right)=H^{*}(Z)$.

Consider the map $p$ from the ring $\mathbb{C}\left[\sigma_{1}, \cdots, \sigma_{g}\right] /\left(\prod_{i}\left(1-x_{i}^{2}\right)=1\right)$ into the ring $\mathbb{C}\left[\tau_{1}, \cdots, \tau_{g-1}\right] /\left(\prod_{j}\left(1-x_{j}^{2}\right)-1\right)$ induced by the map $\mathbb{C}\left[x, \cdots, x_{g}\right] \rightarrow \mathbb{C}\left[x_{2}, \cdots, x_{g}\right]$, with $x_{1} \mapsto 0, x_{2} \mapsto x_{2}, \cdots, x_{g} \mapsto x_{g}$. Under the identifications made just before Lemma $8, p$ is nothing but the restriction map of the previous paragraph. The kernel of $p$ is easily seen to be generated by $\sigma_{g}$.

In the proof of Theorem 3, we need the following lemmata.

Lemma 9. If $k \leq g$, then in the ring $\mathbb{C}\left[\sigma_{1}, \cdots, \sigma_{g}\right] /\left(\prod\left(1-x_{i}^{2}\right)-1\right)$, the product $\sigma_{k}^{2} \sigma_{k+1} \cdots \sigma_{g}$ vanishes. 
Proof. By induction on $g$. Denote by $\beta$ the product $\sigma_{k}^{2} \sigma_{k+1} \cdots \sigma_{g}$. Consider the map $p$ into $\mathbb{C}\left[\tau_{1}, \cdots, \tau_{g-1}\right] /\left(\prod\left(1-x_{j}^{2}\right)-1\right)$. The element $\alpha=\sigma_{k}^{2} \sigma_{k+1} \cdots \sigma_{g-1}$ maps, under $p$, to the element $\tau_{k}^{2} \tau_{k+1} \cdots \tau_{g-1}$ which is zero by induction assumption. Therefore, by Lemma 8 , the element $\alpha$ is divisible by $\sigma_{g}$. Now, $\beta=\alpha \sigma_{g}$ and is thus divisible by $\sigma_{g}^{2}$. However, $\sigma_{g}^{2}$ is the highest degree term in the graded equation $\prod\left(1-x_{i}^{2}\right)=1$ and hence is zero. Therefore, $\beta$ also vanishes.

Lemma 10. The element $\sigma_{1} \sigma_{2} \cdots \sigma_{g}$ is non-zero, i.e. it generates the top degree (degree $g(g+1)$ ) cohomology of $\widehat{X}$.

Proof. By induction on $g$. Since the only class in degree two is $\sigma_{1}$, it is clear that it is the Kahler class of $\widehat{X}$. Hence, the top degree cohomology is generated by $\sigma_{1}^{\frac{g(g+1)}{2}}$. We will prove that $\sigma_{1}^{g(g+1) / 2}$ is a multiple of $\sigma_{1} \cdots \sigma_{g}$. This will prove the lemma.

Now, the cohomology of $Z=\widehat{S p_{2 g-2}} / U(g)$ is the quotient of that of $\widehat{X}$, by the ideal $\sigma_{g}$. Since the dimension of $Z$ is $g(g-1) / 2$, it follows that $\sigma_{1}^{g(g+1) / 2}$ vanishes on $Z$. Thus, in the ring $H^{*}(\widehat{X})$, we have $\sigma_{1}^{(g+1) g / 2}=\sigma_{g} \psi$ where $\psi$ is a degree $g(g-1) / 2$ - element. Now, by induction assumption, the restriction of the element $\psi$ to $Z$ is a multiple of $\sigma_{1} \cdots \sigma_{g-1}$. Consequently, $\psi=\lambda \sigma_{1} \cdots \sigma_{g-1}+\sigma_{g} \phi$ for some $\phi \in H^{*}(\widehat{X})$. Multiplying this last equation by $\sigma_{g}$ and noting that $\sigma_{g}^{2}=0$, we see that $\sigma_{1}^{g(g+1) / 2}=\lambda \sigma_{1} \cdots \sigma_{g}$, proving the lemma.

We will now begin the proof of Theorem 3 .

By the criterion of Theorem 1, it is enough to show that under the Borel map, the image of the compact dual class $[\widehat{Y}]$ is non-zero, where $\widehat{Y}$ is the compact dual of the symmetric space of $H$. We will assume for simplicity that $H$ is a product of two symplectic groups: put $a+b=g$ with $a \geq b$, and set $H=S p_{2 a} \times S p_{2 b} \subset S p_{2 g}$. The proof in the general case is tedious, and we omit it.

Now, the cohomology ring $H^{*}\left(\widehat{S p_{2 a}} / U(a)\right)$ may be identified with $\mathbb{C}\left[x_{1}, \cdots, x_{a}\right]^{S_{a}}$ $/\left(\prod_{i}\left(1-x_{i}^{2}\right)=1\right)$, where $S_{a}$ is the symmetric group on the $a$ letters $x_{1}, \cdots, x_{a}$ and $i$ runs from 1 to $a$. Let $\alpha_{1}, \cdots, \alpha_{a}$ be the elementary symmetric functions in the variables $x_{1}, \cdots, x_{a}$.

Similarly, the cohomology ring $H^{*}\left(\widehat{S p_{2 b}} / U(b)\right)$ may be identified with $\mathbb{C}\left[x_{a+1}, \cdots\right.$, $\left.x_{a+b}=x_{g}\right]^{S_{b}} /\left(\prod_{i}\left(1-x_{i}^{2}\right)=1\right)$, where $S_{b}$ is the symmetric group on the $b$ letters $x_{a+1}, \cdots, x_{a+b}=x_{g}$ and $i$ runs from $a+1$ to $a+b=g$. Let $\beta_{1}, \cdots, \beta_{b}$ be the elementary symmetric functions in the variables $x_{a+1}, \cdots, x_{g}$. 
The top degree term (of degree $\left.2\left(\frac{a(a+1)}{2}+\frac{b(b+1)}{2}\right)\right)$ in the cohomology group of the product $H^{*}\left(\widehat{S p_{2 a}} / U(a) \times \widehat{S p_{2 b}} / U(b)\right)$ is easily seen (by Lemma 10) to be generated by the element $\gamma=\alpha_{1} \cdots \alpha_{a} \otimes \beta_{1} \cdots \beta_{b}$.

The above description of the cohomology of the compact duals of the spaces $S p_{2 g} / U(g), S p_{2 a} / U(a)$ and $S p_{2 b} / U(b)$ identifies them as the rings generated by certain elementary symmetric functions modulo the ideal of relations $\prod_{i}\left(1-x_{i}^{2}\right)=$ 1 for certain integers $i$ (where $i$ runs respectively from 1 to $g, 1$ to $a$ and $a+1$ to $g=a+b)$. From this it is easy to see that the image of $\sigma_{k}$ in the cohomology of the product $\widehat{S p_{2 a}} \times \widehat{S p_{2 b}} / U(a) \times U(b)$ is the sum $\sum_{r} \alpha_{r} \otimes \beta_{k-r}$ where $r$ runs from 0 to $k$ (if $r \geq a+1$ then $\alpha_{r}=0$ by convention). In particular, the image of $\sigma_{g}$ is $\alpha_{a} \otimes \beta_{b}$. By induction on $k$, and by using Lemma 9 applied to the symplectic groups $S p_{2 a}$ and $S p_{2 b}$, one can show that the image of $\sigma_{g} \sigma_{g-2} \cdots \sigma_{g-2 k}$ is

$$
\alpha_{a} \alpha_{a-1} \cdots \alpha_{a-r} \otimes \beta_{b} \beta_{b-1} \cdots \beta_{b-r} .
$$

In particular, if $k=b$ we get that the image of $\sigma_{g} \sigma_{g-2} \cdots \sigma_{g-2 b}$ is the element

$$
\alpha_{a} \alpha_{a-1} \cdots \alpha_{a-b} \otimes \beta_{b} \beta_{b-1} \cdots \beta_{1} .
$$

Thus, the top degree term $\gamma$ (of the cohomology of the product of $\widehat{S p_{2 a}} / U(a)$ and $\left.\widehat{S p_{2 b}} / U(b)\right)$ of the last but one paragraph, lies in the image of the element $\theta=\left(\sigma_{g} \sigma_{g-2} \cdots \sigma_{g-2 b}\right)\left(\sigma_{1} \sigma_{2} \cdots \sigma_{a-b-1}\right)$ under the restriction map of the cohomology of $\widehat{X}$ to the cohomology of the product $\widehat{S p_{2 a}} / U(a) \times \widehat{S p_{2 b}} / U(b)$.

By the definition of the class $[\widehat{Y}](\widehat{Y}$ being the compact dual of the symmetric space associated to $\left.S p_{2 a} \times S p_{2 b}\right)$, this implies the equality $\theta \wedge[\widehat{Y}]=\sigma_{1} \cdots \sigma_{g}$, namely a generator of the top degree (of degree $2\left(\frac{g(g+1}{2}\right)$ ) cohomology of $\widehat{X}$. Since this wedge product is non-zero, and $\theta$ is divisible by $\sigma_{g}$, it follows that the class $[\widehat{Y}]$ is not orthogonal to the ideal generated by $\sigma_{g}$.

Now, Lemma 8 implies that $[\widehat{Y}]$ is not in the kernel of the Borel map. This proves Theorem 3 .

\section{The Unitary Group}

In this section, $G=U(p, q)$ will denote the unitary group in $n=p+q$ variables, with $1 \leq p \leq q$. The $\mathbb{Q}$ structure is defined as follows. Let $V$ be an $n$-dimensional 
vector space over an imaginary quadratic extension $E$ over $\mathbb{Q}$. Consider the $E$ valued Hermitian form in $n$ variables given by

$$
h(v, v)=\sum_{i=1}^{i=p}\left|z_{i}\right|^{2}-\sum_{i=p+1}^{i=n}\left|z_{i}\right|^{2}
$$

with $v=\left(z_{1}, \cdots, z_{n}\right) \in V$. The group preserving this Hermitian form is a $Q$ algebraic group and the group of its real points is the group $U(p, q)$.

The group $K=U(p) \times U(q)$ is a maximal compact subgroup of $U(p, q)$ and the group $T$ of diagonals in $K$ is a maximal torus of $G$ and $K$. As in section (4.1), if $\widehat{X}$ is the compact dual of $G / K(\widehat{X}$ is the Grassmanian of $p$ planes in $n$ dimensional complex vector space), then the cohomology of $\widehat{X}$ may be identified with the quotient ring (see $[\mathrm{M}-\mathrm{T}]$ )

$$
\frac{\mathbb{C}\left[x_{1}, \cdots, x_{p} ; y_{i}, \cdots, y_{q}\right]^{S_{p} \times S_{q}}}{\prod\left(1+x_{i}\right) \prod\left(1+y_{j}\right)=1}
$$

In this equality, $S_{p}$ (resp, $S_{q}$ ) is the permutation group of the $p$ letters $x_{1}, \cdots, x_{p}$ (resp. the $q$ letters $\left.y_{1}, \cdots, y_{q}\right)$. The superscript denotes the ring of invariants under the product group $S_{p} \times S_{q}$. The variable $i$ (resp, $j$ ) runs from 1 to $p$ (resp. 1 to $q)$. The equation $\prod\left(1+x_{i}\right) \prod\left(1+y_{j}\right)=1$ is a graded equation.

Let $\sigma_{1}, \cdots, \sigma_{p}$ (resp. $\tau_{1}, \cdots, \tau_{q}$ ) denote the elementary symmetric functions in the variables $x_{1}, \cdots, x_{p}$ (resp. $y_{1}, \cdots, y_{q}$ ). Then, the cohomology of $\widehat{X}$ is the ring

$$
\frac{\mathbb{C}\left[\sigma_{1}, \cdots, \sigma_{p} ; \tau_{1}, \cdots, \tau_{q}\right]}{\left(1+\sigma_{1}+\cdots+\sigma_{p}\right)\left(1+\tau_{1}+\cdots+\tau_{q}\right)=1}
$$

where as before, the equation involving $\sigma$ 's and $\tau$ 's is a graded one.

Lemma 11. With the foregoing notation, the kernel of the Borel map for $G=$ $U(p, q)$ is the orthogonal complement of the ideal generated by $\sigma_{p}$ and $\tau_{q}$ in the cohomology ring $H^{*}(\widehat{X})$.

Proof. As $p \leq q$, the $\mathbb{Q}$-rank of the group $G=U(p, q)$ is $p$. The Hermitian form $h$ defining $G$ is a direct sum of $p$ "hyperbolic" (i.e. isotropic over $\mathbb{Q}$ ) Hermitian forms $h_{1} \oplus \cdots \oplus h_{p}$ and an anisotropic hermitian form $h_{0}$. It is then easy to see that a Levi subgroup is of the form $G L_{k} \times U(p-k, q-k)$ for some $k \leq p$. Thus, $\widehat{X_{L}}$ is the product of the symmetric spaces $S U(k) / S O(k)$ and $U(p+q-2 k) /(U(p-k) \times U(q-k))$.

An argument involving Chern classes of homogeneous vector bundles similar to that in the proof of Lemma 7 shows that all the (positive degree) cohomology 
classes on $U(2 k) / U(k) \times U(k)$ vanish on $i(S U(k)) / S O(k)$, for any embedding $i$ of $S U(k)$ in $U(2 k)$ such that the intersection $i(S U(k)) \cap(U(k) \times U(k))=S O(k)$ and $i$ is the identity on $S O(k)$. In particular, it shows (cf. the paragraph preceding Lemma 8) that the kernel of the map

$$
H^{*}(\widehat{X}) \rightarrow \prod H^{*}\left(\widehat{X}_{L}\right)
$$

(where the product is over all the standard Levi subgroups as before), is the kernel of the map

$$
H^{*}(\widehat{X}) \rightarrow H^{*}(U(p+q-2) /(U(p-1) \times U(q-1))) .
$$

The latter kernel may easily be shown to be the ideal generated by $\sigma_{p}$ and $\tau_{q}$.

By Franke, the kernel of the Borel map is precisely the orthogonal complement of this ideal. Hence the Lemma.

Lemma 12. With the previous notation, the element $\tau_{q}^{p}$ generates the top degree cohomology (in degree $2 p q$ ) of $\widehat{X}$.

Proof. By induction on $p$.

Consider the restriction map from $H^{*}(\widehat{X})$ to $H^{*}\left(\widehat{Y}_{1}\right)$, where $\widehat{Y}_{1}$ is the compact dual of the symmetric space associated to $U(p-1, q) \subset U(p, q)$. The kernel of this map is generated by $\sigma_{p}$, as may be easily seen. In particular, if $R_{p q-p-q}$ be the graded piece of the cohomology ring $R$ of $\widehat{X}$ (since $H^{(p-1) q}\left(\widehat{Y}_{1}\right)$ is one dimensional) it follows that $\sigma_{p} R_{p q-p-q}$ is of codimension one in $H^{(p-1) q}(\widehat{X})$. But, wedging by $\tau_{q}$ kills this subspace (since, by the graded relation, $\tau_{q} \sigma_{p}=0$ ). By definition, wedging by the cycle class $\left[\widehat{Y}_{1}\right]$ also kills this subspace. Therefore, $\left[\widehat{Y}_{1}\right]=\lambda \tau_{q}$ for some non-zero scalar $\lambda$.

By induction, the restriction of $\tau_{q}^{p-1}$ generates the top degree cohomology of $\widehat{Y}_{1}$. By the definition of the cycle class $\left[\widehat{Y}_{1}\right], \tau_{q}^{p-1}\left[\widehat{Y}_{1}\right]$ is non-zero, i.e. generates the top degree cohomology of $\widehat{X}$. By the previous paragraph, this element is, up to non-zero scalars, $\tau_{q}^{p}$.

Remark. Note that since $\sigma_{1}$ is the Kahler class on $\widehat{X}$ and restricts to a Kahler class on $\widehat{Y}_{1}$, we have also proved that $\tau_{q}\left(\sigma_{1}\right)^{(p-1) q}=\tau_{q}^{p} \neq 0$.

We now begin the proof of Theorem 4 .

5.1. Part 1 of Theorem 4. The Hermitian space $V$ may be split into a direct sum of Hermitian spaces $V_{i}$ and $W$, where, on each $V_{i}$, the form $h$ is of type $\left(p_{i}, q_{i}\right)$; on $W$, it is of type $\left(0, q-\sum q_{i}\right)$. Then $\sum p_{i}=p$ and $\sum q_{i} \leq q$. We thus 
get an embedding of $H=\prod U\left(p_{i}, q_{i}\right)$ in $U(p, q)$.

Let $\xi_{1}, \cdots, \xi_{l}$ (resp. $\left.\omega_{1}, \cdots, \omega_{l}\right)$ denote the analogues of $\tau_{q}$ (resp. of $\sigma_{1}$ ) for the groups $U\left(p_{1}, q_{1}\right), \cdots, U\left(p_{l}, q_{l}\right)$ and $Z=Z_{1} \times \cdots Z_{l}$ be the products of the compact symmetric spaces associted to the product group. The restriction of $\tau_{q}$ to this product variety $Z$ is clearly the tensor product $\xi_{1} \otimes \cdots \otimes \xi_{l}$. Here we use the fact that $\sum p_{i}=p$. An easy computation shows that if $d\left(\operatorname{resp} d_{i}\right)$ is the dimension of $Z$ (resp. $Z_{i}$ ) then the restriction of the element $\tau_{q} \sigma_{1}^{d}$ to the product variety $Z$ is the tensor product $\xi_{1} \omega_{1}^{d_{1}} \otimes \cdots \otimes \xi_{l} \omega_{l}^{d_{l}}$. By Remark 5 applied to $U\left(p_{i}, q_{i}\right)$ for each $i$, we get that this tensor product element is the top degree cohomology class of $Z$. Thus, $\tau_{q} \sigma_{1}^{d} \wedge[Z] \neq 0$. Therefore, the cycle class of the compact dual associated to $\prod U\left(p_{i}, q_{i}\right)$ is not orthogonal to an element of $\tau_{q} R$. In particular (from Lemma 11), $[Z]$ does not lie in the kernel of the Borel map. This proves, by Theorem 1 , that the modular symbol $\left[S_{H}(\Gamma)\right]$ is non-zero.

5.2. Part (2) of Theorem 4. The cohomology of the compact dual $\widehat{Y}$ associated to $S p_{2 g}$ is (see Lemma 6)

$$
\frac{\mathbb{C}\left[\sigma_{1}, \cdots, \sigma_{g}\right]}{\prod\left(1-x_{i}^{2}\right)=1}
$$

The cohomology of $\widehat{X}$ for the group $U(g, g)$ is

$$
\frac{\mathbb{C}\left[\sigma_{1}, \cdots, \sigma_{g} ; \tau_{1}, \cdots, \tau_{g}\right]}{\prod\left(1+x_{i}\right)\left(1+y_{i}\right)=1}
$$

The restriction map from the cohomology of $\widehat{X}$ to that of $\widehat{Y}$, is induced by $x_{i} \mapsto x_{i}$ and $y_{i} \mapsto-x_{i}$. Therefore, the top degree class $\sigma_{1} \cdots \sigma_{g}$ of $\widehat{Y}$ is in the image of the product $\tau_{1} \cdots \tau_{g}$. Hence, $[\widehat{Y}] \wedge \tau_{1} \cdots \tau_{g}$ generates the top degree class of $\widehat{X}$. This means that the cycle class $[\widehat{Y}]$ is not orthogonal to the ideal generated by $\tau_{g}$. Thus, by Lemma 11, $[\widehat{Y}]$ is not in the kernel of the Borel map, whence, by Theorem 1, the modular symbol $\left[S_{H}(\Gamma)\right]$ is non-zero.

5.3. Proof of Theorem 5. The Hermitian space $(E, h)$ is such that for the associated group $G$, we have $G(\mathbb{R})=S U(1, q)$. We first prove the following lemma.

Lemma 13. If $\alpha$ is a non-zero holomorphic 1-form on $S(\Gamma), \omega$ is the Kahler class on $\widehat{X}$ and $j$ the Borel map, then the cup-product $\alpha \wedge j(\omega)$ is non-zero.

Proof. It is enough to prove (since $\omega$ is $G\left(\mathbb{A}_{f}\right)$ - invariant), that for some $g \in$ $G\left(\mathbb{A}_{f}\right)$, the cup-product $g(\alpha) \wedge \omega$ is non-zero. Consider the three dimensional hermitian subspace $\left(F,\left.h\right|_{F}\right)$ of the Hermitian space $(V, h)$, (a vector space over 
the field $E$ ) where the Hermitian form does not represent a zero. By weak approximation, this is possible (since one may locate a three dimensional subspace over $p$-adic field $E_{v}$ of the hermitian vector space $V \otimes E_{v}, h \otimes E_{v}$ where the Hermitian form does not represent a zero).

This is an anisotropic subspace, whence the associated group $H_{0}=S U(F, h)$ is anisotropic over $\mathbb{Q}$ and is isomorphic to $S U(1,2)$ over $\mathbb{R}$. Thus, the subvariety $S_{H_{0}}(\Gamma)$ is compact. If $\alpha$ is a non-zero holomorphic 1-form on $S(\Gamma)$ class on $\widehat{X}$, then there exists a $g \in G\left(\mathbb{A}_{f}\right)$ such that the restriction of the form $g^{*}(\alpha)$ to $S_{H_{0}}(\Gamma)$ is non-zero. By replacing $\alpha$ by $g^{*}(\alpha)$, we may assume that $g=1$. However, since $S_{H_{0}}(\Gamma)$ is compact, this means that the restriction of $\alpha \wedge \bar{\alpha} \wedge j(\omega)$ to $S_{H_{0}}(\Gamma)$ is non-zero. In particular, $\alpha \wedge j(\omega) \neq 0$.

In the above, $\bar{\alpha}$ denotes the anti-holomorphic 1-form which is the complex conjugate of the holomorphic form $\alpha$. The complex conjugation is on the cohomology group $H^{1}(S(\Gamma), \mathbb{C})=H^{1}(S(\Gamma), \mathbb{R}) \otimes \mathbb{C}$.

Proof. of Theorem 5. We will argue by contradiction. Suppose that for $H=$ $S U(1, q-1)$ the cycle class $\left[S_{H}(\Gamma)\right]$ is always (i.e. for every $\left.\Gamma\right) G\left(\mathbb{A}_{f}\right)$-invariant. By Theorem 1, this class is then equal to $j([\widehat{Y}])$. However, $H^{2}(\widehat{X})$ is one dimensional $\left(\widehat{X}=\mathbf{P}^{q}\right.$, the complex projective $q$-space). Hence $[\widehat{Y}]=\omega$ the Kahler class.

If $\alpha$ is a non-zero holomorphic form on $S(\Gamma)$ (such forms exist by $[\mathrm{K}]$ ), then $\alpha \wedge j([\widehat{Y}]) \neq 0$ by Lemma 13. Thus, the restriction of $\alpha$ to $S_{H}(\Gamma)$ does not vanish, for every $\Gamma$. Thus, at the level of $H^{1}$ the restriction map from $S U(1, q)$ to $S U(1, q-1)$ is injective for every $\Gamma$. In particular, the holomorphic cohomology classes of degree one associated to $S U(n, 1)$ restrict injectively to those on $S U(n-$ $1,1)$.

We now recall the criterion of [V2], for the span of Hecke-translates of $\left[S_{H}(\Gamma]\right.$ to be infinite dimensional. In the statement of the following theorem, the map Res refers to an "Oda style" restriction map.

Theorem 14. (see [V2], Theorem 1). Suppose that $G$ is almost $\mathbb{Q}$-simple and that

1. The centralizer $Z_{G}(H) \cap K$ is not contained in the center $Z(G)$ of $G$.

2. For some integer $m \leq d=\operatorname{dim}\left(S_{H}(\Gamma)\right)$ (dimension as a complex manifold), the restriction map

$$
\text { Res }: H^{m, 0}(S(\Gamma)) \rightarrow \prod_{g \in G(\mathbb{Q})} H^{m, 0}\left(S_{H}\left(g \Gamma g^{-1}\right)\right)
$$


is non-zero.

Then, there exists a congruence subgroup $\Gamma^{\prime}$ of $\Gamma$ such that the cycle class $\left[S_{H}\left(\Gamma^{\prime}\right)\right]$ is not $G\left(\mathbb{A}_{f}\right)$ invariant.

For the group $G$ with $G(\mathbb{R})=S U(n, 1)$ up to compact factors there exist elements in $G(\mathbb{Q})$ which centralize $S U(n-1,1)$ but do not lie in the center of $G$, since the centralizer of $S U(n-1,1)$ is the group $U(1)$ (all these viewed, by restriction of scalars, as groups over $\mathbb{Q}$ ).

Thus, the conditions of Theorem 1 of [V2] are satisfied and so by Theorem 1 of [V2], there exists a $\Gamma$ so that the cycle class $\left[S_{H}(\Gamma)\right]$ is not $G\left(\mathbb{A}_{f}\right)$-invariant, which contradicts our assumption.

Acknowledgement. This work was begun when both the authors were visiting MPI, Bonn; the hospitality of MPI is gratefully acknowledged. The authors also thank Arvind Nair for very helpful conversations. Birgit Speh was partially supported by the NSF grant DMS-007056.

The second named author considers it one of the great privileges of his life to have met Professor Armand Borel. We dedicate this paper to his memory. We thank Professor Lizhen Ji for his kind invitation to contribution to this volume.

\section{REFERENCES}

[A] A.Ash., Non square integrable cohomology of arithmetic groups, Duke Math. Journal 47 (1980) 435-449.

[A-B] A.Ash and A.Borel, Generalized modular symbols, Lecture Notes in Mathematics 1447, Springer-Verlag (1990), $57-75$.

[A-G-R] A.Ash, D.Ginzburg and S.Rallis, Vanishing periods of cusp forms over modular symbols, Math. Annalen 296 (1993), 709 - 723.

[F] J.Franke, A Topological Model for Some Summand of the Eisenstein Cohomology of Congruence Subgroups, Preprint, Bielefeld University, 1991.

[K] D.Kazhdan, Some applications of the Weil representation, Journal d'analyse, 32, 233248 (1977).

[M-T] Mimura and Toda, Topology of Lie Groups (I) and (II), Translations of Math. Monographs, Vol 91, AMer. Math. Soc. (1991).

[H1] G.Harder, Eisenstein cohomology of arithmetic groups. The case $G L_{2}$. Invent. Math 89, $37-118,(1987)$

[H2] G.Harder, Modular symbols and special values of L-functions, available at ftp://ftp.math.uni-bonn.de/people/harder/Eisenstein/Modsym.pdf

[R-S] J.Rohlfs and B.Speh, Pseudo-eisenstein Forms and the cohomology of arithmetic groups, to appear in the proceedings of the conference in honor of Ragunathan, Tata Institute 2002 .

[V] T.N.Venkataramana, Cohomology of compact locally symmetric spaces, Compositio.Math 125, 221-253, (2001). 
[V2] T.N.Venkataramana, On Cycles on Compact Shimura varieties. Monatshefte Mathematik 135, 221-244 (2002).

B. Speh

Department of Mathematics, 310 Malott Hall

Cornell University, Ithaca N.Y.14853-4201, U.S.A

E-mail: speh@math.cornell.edu

T. N. Venkataramana

School of Mathematics, Tata Institute of Fundamental Research

Homi Bhabha Road, Bombay - 400 005, INDIA.

E-mail: venky@math.tifr.res.in 